\title{
Magnetic resonance imaging in paediatrics
}

Magnetic resonance imaging (MRI) has been available in clinical practice since the early 1980 s. From the start MRI was attractive because of the safety of the technique when compared to $x$ ray imaging, as well as the anatomical detail and pathological contrast seen on the images. By the end of the decade MRI had evolved into a useful method for the evaluation of the central nervous and musculoskeletal systems. The number of machines available in the UK was beginning to increase, although the number per head of population has always remained behind that of the USA, Japan, and most European countries.

By the end of 1992, the Royal College of Radiologists reported that there were 90 fixed site machines for MRI in the UK. ${ }^{1}$ The college recommended an increase to 250 machines (four per million population) in order to cope with the increased demand for central nervous system and musculoskeletal MRI. While the report of the Royal College of Radiologists acknowledged new indications and methodologies for MRI, it is clear with hindsight the authors underestimated the current renaissance in MRI.

The key to this is better hardware for MRI. Magnet technology is now universally good. Important recent technical advances have been in gradient coil power, design, and stability. This has allowed faster and faster switching of stronger and stronger gradient fields.

There are many methods to obtain a magnetic resonance signal from a patient. All of these involve the use of radiofrequency pulses which perturb the induced resting magnetisation in the patient's body water within the strong external magnetic field. In the 1980s the workhorse pulse sequences were so-called 'spin echo', which involved the use of both $90^{\circ}$ and $180^{\circ}$ radiofrequency pulses. A $90^{\circ}$ pulse will rotate the magnetic field through $90^{\circ} \mathrm{s}$ into the transverse plane, while a $180^{\circ}$ pulse will reverse the direction of the magnetic vector. Although robust and informative the spin echo sequences are slow. Today many have been replaced by gradient echo sequences, which can be used to improve anatomic resolution and/or reduce imaging time. In gradient echo sequences the magnetic vector may be rotated through a reduced angle (even $1-2^{\circ} \mathrm{s}$ is possible) with considerable improvement in image acquisition time. One particular advance has been in the use of gradient echo pulse sequences to rapidly acquire multiple single slices. These can be repeated at the same anatomic level to provide a cine effect, for example the beating heart, or can be taken serially at different levels to cover an anatomic region in a short time. In this mode magnetic resonance is working like a computed tomographic scanner. Each slice is acquired in perhaps one third second and repeatable every second. An individual scan can be repeated if degraded by patient movement. With the older spin echo technology a single patient movement could disrupt the entire set of images collected over 12 minutes.

The fast gradient echo acquisitions can be gated with the ECG and pulse oximeter. ${ }^{2}$ They may be acquired in two dimensions like computed tomograms or in three dimensions allowing image reconstruction at a later time along any anatomical plane. Other improvements in postprocessing of images has led to magnetic resonance angiography where a signal from the blood is highlighted, while background signal is reduced. The computer can use these sequences to display a 'road map' of the arterial or venous system that compares very favourably with conventional angiographic techniques. Quantitation of flow data is also possible.

These improvements in MRI have only increased the demand for scans. The cardiovascular system, chest, abdomen, and pelvis are now realistic indications for examinations. In the cardiovascular system magnetic resonance interfaces with recent developments in ultrasound. It has become clear that MRI should be the first and only investigation for a number of cardiovascular pathologies, for example aortic thrombosis. In the chest, abdomen, and pelvis MRI is used as a complementary test to ultrasound and computed tomography, but it is also quite clear that computed tomography is increasingly requested because of problems with access to MRI, rather than because it remains the better technique.

All this should be good news to those looking after children. It is not difficult to imagine the ideal paediatric radiology department consisting of ultrasound and MRI, backed up with plain radiography and nuclear medicine as appropriate. Yet these tremendous advances in MRI are not yet impacting on paediatric radiological practice in the manner they should. Some of the reasons for this include the hostile environment of MRI, the formidable technology, a lack of resources, and a lack of published evidence to show the value of MRI in children.

The MRI examination requires the patient to lie still on a couch within the narrow bore of a large magnet for 15 or more minutes. Up to $10 \%$ of alert adults find this impossible, and many more find the MRI examination stressful. For children it is even more difficult. In practice recently fed babies can usually be examined while asleep and older children can be talked through the examination by a parent or radiographer. Most children, especially those who are very ill or disturbed, will require sedation or anaesthesia. This is the true risk of the examination, not the MRI procedure itself. It is against the risk of sedation or anaesthesia that the benefit of the examination must be judged.

The joint Royal College of Radiologists/Royal College of Anaesthetists report into sedation and anaesthesia warns that sedation cannot easily be separated from anaesthesia and that when sedation is given, the same support as that necessary for anaesthesia should be available. ${ }^{3}$ Yet few, if any, UK MRI units have a paediatric anaesthetist and paramedical team and MRI compatible anaesthetic and monitoring equipment freely available. In many centres a lack of these resources can be linked with the use of sedation without the recommended back-up cover. The risks can be minimised but not eliminated by the involvement of paediatricians and anaesthetists together with careful protocols. Many MRI units undertaking paediatric sedation lack even modest monitoring equipment such as a pulse oximeter (G R Cherryman, unpublished audit on UK MRI centres, 1993).

The MRI environment is also hostile to children as it is almost always located in and designed for the adult world. The NHS does not have the resources to demand and purchase better, specifically designed paediatric hardware, so manufacturers will not build it.

MRI represents a formidable challenge to the radiologist. Several years' immersion in the method are required to become completely familiar with the technology, as well as the options, artefacts, problems, etc. Most working paediatric radiologists do not have the time or the referrals 
to gain this experience. In many centres MRI is performed by technique rather than system based radiologists. This is likely to continue for some years while MRI continues to develop at its present pace. It is easy to recommend cooperation between paediatric radiologists and those experienced in MRI rather than a head to head turf battle, but as long as radiologists remain jealous of their expertise and patients, paediatric radiologists will remain disadvantaged in learning MRI. The arrival of an MRI unit almost always imposes an additional workload on the radiologists and many departments find it difficult to cover and fund double consultant MRI sessions.

The lack of financial resources has other effects. MRI units are universally busy with long waiting lists and high overheads. Paediatric scanning is time consuming, difficult, and expensive. There are huge pressures on the staff to increase the throughput of adult patients. In some instances it is the paediatric service that will suffer.

Finally, the paediatrician needs to know what MRI can achieve. How to make the risk/benefit analysis for each individual. How to justify the purchase of expensive scans, equipment, and expertise from a limited budget. Unfortunately, because of the difficulties surrounding paediatric MRI in many instances this evidence is not available and cannot become available until there is better and safer access to MRI for children. Clinicians have to rely on their own, often restricted, experience with MRI, rather than hard data.

Where there is information, especially in the central nervous, musculoskeletal, and cardiovascular systems the results have been encouraging. MRI should be considered of value in the brain and recent studies have reported its use in neoplasia, ${ }^{4}$ brain development, ${ }^{5}$ cerebral injury and suspected abuse, ${ }^{6}$ and also magnetic resonance angiography ${ }^{7}$ and cerebral perfusion. ${ }^{8}$ Other studies have reported on MRI of the spine for injury, ${ }^{9}$ metastases, ${ }^{10}$ and scoliosis $^{11}$ and for the evaluation of musculoskeletal tumours $^{12}$ and other musculoskeletal abnormalities. ${ }^{13}$ In the trunk MRI is mainly used for solid organ imaging ${ }^{14} 15$ and for the evaluation of posterior mediastinal masses. ${ }^{1617}$ In the management of congenital heart disease MRI provides additional information to ultrasound and may obviate the need for angiography..$^{18-20}$

It is clear what is needed. Child friendly MRI units, safe sedation and anaesthesia, committed and cooperative paediatric radiologists, clinician and parent pressure for resources, and an affirmation of the need to collect and report on paediatric MRI data. In order to speed this up a centralised, multicentre study of the indications, risks, and value of MRI in children should be established.

In paediatric radiological practice MRI is likely to complement other diagnostic tests, to a greater extent than in adults because of the risks associated with sedation and anaesthesia. Second line must not be allowed to mean second rate.

Department of Radiology,

G R CHERRYMAN

Faculty of Medicine,

Leicester Royal Infirmary,

Leicester LE1 $5 \mathrm{WW}$

1 Report of a Royal College of Radiologists Working Party. Provision of magnetic resonance imaging services in the United Kingdom. London: Royal College of Radiologists, 1992.

2 Denslow S, Buckles DS. Pulse oximetry-gated acquisition of cardiac MR images in patients with congenital cardiac abnormalities. $A \mathcal{F} R$ 1993; 160: 831-3.

3 Report of a Joint Royal College of Anaesthetists and Royal College of Radiologists Working Party. Sedation and anaesthesia in radiology. London Royal College of Anaesthetists/Royal College of Radiologists, 1992.

4 Tice HM, Jones KM, Mulkern RV, et al. Fast spin-echo imaging of intracranial neoplasms. F Comput Assist Tomogr 1993; 17: 425-31.

5 Grodd W. Normal and abnormal patterns of myelin development of the fetal and infantile human brain using magnetic resonance imaging. Curr Opin Neurol Neurosurg 1993; 6: 393-7.

6 Sklar ML, Quencer RM, Bowen BC, Altman N, Villanueva PA. Magnetic resonance applications in cerebral injury. Radiol Clin North $\mathrm{Am}$ 1992; 30: 353-66.

7 Ostertun B, Solymosi L. Magnetic resonance angiography of cerebral developmental venous abnormalities: its role in differential diagnosis. developmental venous abnorma

8 Tzika AA, Massoth RI, Ball WS Jr, Majumdar S, Dunn RS, Kirks DR Cerebral perfusion in children: detection with dynamic contrast-enhanced T2^ weighted MR images. Radiology 1993; 187: 449-58.

9 Davis PC, Reisner A, Hudgins PA, Davis WE, O'Brien MS. Spinal injuries in children: role of MR. Am $\mathcal{F}$ Neuroradiol 1993; 14: 607-17.

10 Tanabe $M$, Takahashi $H$, Ohnuma N, Iwai J, Yoshida H. Evaluation of bone marrow metastasis of neuroblastoma and changes after chemotherapy by MRI. Med Pediatr Oncol 1993; 21: 54-9.

11 Barnes PD, Brody JD, Jaramillo D, Akbar JU, Emans JB. Atypical idiopathic scoliosis: MR imaging evaluation. Radiology 1993; 186: 247-53.

12 Greenfield GB, Arrington JA, Kudryk BT. MRI of soft tissue tumours. Skeletal Radiol 1993; 22: 77-84.

13 Schlesinger AE, Hernandez RJ. Diseases of the musculoskeletal system in children: imaging with CT, sonography and MR. $A \mathcal{F} R$ 1992; 158: 729-41. 14 Vade A, Azienstein R. Magnetic resonance imaging of abdominal masses in children. F Pediatr Surg 1993; 28: 82-8.

15 Lumkin B, Anderson MW, Ablin DS, McGahan JP. CT, MRI and color Doppler ultrasound correlation of pancreatoblastoma: a case report. Pediatr Radiol 1993; 23: 61-2.

16 Merton DF. Diagnostic imaging of mediastinal masses in children. AfR 1992; 158: 825-32.

17 Saenz NC, Schnitzer JJ, Eraklis AE, et al. Posterior mediastinal masses. 7 Pediatr Surg 1993; 28: 172-6.

18 Simpson IA, Valdes-Cruz LM, Berthoty DP, et al. Cine magnetic resonance imaging and colour Doppler flow mapping in infants and children with imaging and colour Doppler flow mapping in infants and

19 Bank ER. Magnetic resonance of congenital cardiovascular disease: an update. Radiol Clin North Am 1993; 31: 553-72.

20 Rebergen SA, Ottenkamp J, Doornbos J, Van de Wall EE, Chin JGJ, De Roos A. Postoperative pulmonary flow dynamics after Fontan surgery: assessment with magnetic resonance velocity mapping. $7 \mathrm{Am}$ Coll Cardiol 1993; 21: 123-31. 